\title{
Context- and subgroup-specific language changes in individuals who develop PTSD after trauma.
}

\author{
German Todorov $^{1 *}$, Karthikeyan Mayilvahanan ${ }^{2}$, Christopher K Cain ${ }^{1,3}$, and Catarina Cunha ${ }^{1}$ \\ ${ }^{1}$ Emotional Brain Institute, Nathan Kline Institute, Orangeburg, NY, USA \\ ${ }^{2}$ Department of Neurobiology and Behavior, Stony Brook University School of Medicine, Stony Brook, NY, USA \\ ${ }^{3}$ NYU School of Medicine, Dept. of Child \& Adolescent Psychiatry,1 Park Avenue, 8th Floor, New York, NY 10016, United \\ States \\ *Correspondence: German Todorov, Emotional Brain Institute, Nathan Kline Institute, Orangeburg, NY 10962, USA. E- \\ mail: german.todorov@nki.rfmh.org
}

According to the World Health Organization, mental illness is the leading cause of disability worldwide accounting for $37 \%$ of years of healthy life lost. Early detection and prevention of mental health disorders is important for social stability and economic prosperity of every country and the world at large. Post-traumatic Stress Disorder (PTSD) is a very common condition with more than 3 million cases per year in the US alone. PTSD symptoms usually start soon after a traumatic event. The right diagnosis in a timely manner is key to ensuring prompt treatment that could lead to a full recovery. Unfortunately, social stigma, self-presentation and self-assessment biases often prevent individuals from seeking timely evaluation, leading to delays in treatment and suboptimal outcomes. Previous studies show that various mental health conditions are associated with distinct patterns of language use. Analyzing language use may also help to avoid response bias in self-reports. In this study we show a number of language use differences between PTSD sufferers and controls. Our data suggest that subgroups of people with the same mental health disorder (PTSD in this study) may have salient differences in their language use, particularly in word usage frequencies. Additionally, we show that word usage patterns may vary depending on the type of the text analyzed. These factors must be accounted for when creating screening tools based on language analysis. If properly developed, such tools may facilitate earlier PTSD diagnosis, leading to timely support and treatment, which are associated with better outcomes. 


\section{Introduction}

In recent decades, there has been a dramatic increase in severe mental illness especially in the United States. Since 1955 the percentage of people disabled by mental health disorders has increased five-fold. In the US alone, there are currently almost 6 million people disabled by mental illness. This number rises each day by more than 400 . This is not just a health crisis but also an economic one: direct and indirect global economic costs of mental illness were estimated at US $\$ 2.5$ trillion as of 2010. As reported by the National Center for Post-Traumatic Stress Disorder (PTSD), 7-8\% of the US population will experience PTSD at some point in their lifetime. To maintain economic and social stability, mental illness must be prevented and treated. In the past few decades, considerable progress has been made in understanding mental illness (Norquist and Hyman, 1999), leading to the development of more effective treatment options. Nonetheless a large portion of this population remains untreated. Nationwide epidemiologic studies show that over half of the people with mental health disorders who would benefit from treatment do not receive it (Regier et al., 1993; Kessler et al., 2001).

Numerous studies have tried to explain why some people with mental illness seek treatment while others do not. One common factor is a fear of stigmatization, which is a serious issue in both military and civilian populations (Olfson et al., 1998; Perlick et al., 2001; Link et al., 2008; Ahmedani, 2011; Bonfils et al., 2018). Another important factor is the inability of the affected individuals to recognize the symptoms (Ahmedani, 2011), which prevents help-seeking and at best delays diagnosis and treatment. Most mental health disorders are much easier to control and reverse if they are diagnosed and treated early. Hence delayed diagnosis often means reduced quality of life not only until the time of late treatment but potentially long thereafter. Currently used mental health evaluations such as the DSM (Diagnostic and Statistical Manual of Mental Disorders), demand a well-trained specialist, who is not always promptly available. However, early and accurate diagnosis is key to facilitating timely and effective treatment that could lead to a full recovery (Acosta et al., 2013; Burnam et al., 2014; Brahm et al., 2016; Jones et al., 2017; Horn and Feder, 2018). In that context, individuals with mental health concerns could benefit from an anonymous and easily accessible self-testing tool that analyzes language usage patterns and correlates them to mental health conditions or risk thereof. While unsuitable for any definitive diagnosis, such a tool may prompt at-risk individuals to seek timely mental health evaluation and care. 
In fact, the approach to access mental states by analyzing patterns of language usage is supported by a growing body of research in the fields of psychology and neuroscience (Pennebaker et al., 2003). Previous studies using Linguistic Inquiry and Word Count (LIWC) have found that it is possible to characterize depression and other mental states through analyzing natural language use (Tausczik and Pennebaker, 2010; Booker et al., 2018; Kaplow et al., 2018; Tackman et al., 2018). For example, Stirman and Pennebaker showed that suicidal poets displayed a higher usage of first person pronouns in their texts and less first-plural pronouns than non-suicidal poets (Stirman and Pennebaker, 2001). Rude, Gortner, and Pennebaker demonstrated that the language of depressed students has a higher frequency of first person singular pronouns and negative emotion words, and a lower frequency of positive emotion words, compared to students that never experienced depression (Rude et al., 2004). Together, these results support the social engagement/disengagement model of depression (Fisher and Chon, 1989), and Pyszcynski and Greenberg's self-awareness theory of depression (Pyszczynski and Greenberg, 1987). Furthermore, clinicians have observed higher use of first singular pronouns in depressed patients (Bucci and Freedman, 1981; Weintraub et al., 1981), and lower second-person pronouns usage, which may reflect a decreased sense of community (Simmons et al., 2005).

People with PTSD were shown to use significantly fewer second person pronouns (Coppersmith et al., 2014b). Additionally, studies have shown differences in the use of causal and cognitive words during recalls of negative events (Boals and Klein, 2005), which may be an indicator of the attempt to rationalize and resolve traumatic experiences (Kross and Ayduk, 2008). The analysis of additional linguistic markers, such as the use of negative emotion words, cognition words, and insight words, predicted the future mental health of college students who wrote about traumatic events (Pennebaker, 2001). Furthermore, the presence of words relating to death and dying was an indicator of treatment-resistant PTSD (Alvarez-Conrad et al., 2001). Consequently, the analysis of linguistic elements in different text types could be crucial for understanding cognitive mechanisms associated with trauma and may hold a valuable potential to diagnose and predict PTSD symptoms and subtypes. If properly developed, such technology could help individuals to self-test and public health organizations to screen for possible mental health conditions and prompt further evaluation when warranted, potentially preventing disorders from becoming chronic, debilitating and difficult to treat. 
While language analysis programs could be an effective means for tentative self-diagnosis and mental health screening, their development is impeded by the gaps and inconsistencies in our current understanding of the links between language usage and mental illness. The few existing language analysis programs are based on data from generalized mentally ill populations that commingle otherwise fairly distinct subgroups. This often results in disregarding salient individual differences and may lead to misdiagnosis.

A major hurdle for developing more specific and reliable language based diagnostic tools has been the lack of variety in the available language data. With the growth of social media, internet forums are shown to present a useful source of naturalistic writing from people that use these forums as an anonymous and inexpensive self-help tool, especially for stigmatizing mental health illnesses such as PTSD (Baker et al., 2003; Lampe et al., 2003; Berger et al., 2005). Recent studies show that using an automated word counting approach is an efficient way to characterize the language of online groups (Lyons et al., 2006). Patterns of emotional and cognitive expression in Internet support groups were used to research depression and other affective disorders (Houston et al., 2002; Griffiths et al., 2009), and as a predictor of future mental health in cancer patients and recovering anorexics (Lieberman and Goldstein, 2006; Lyons et al., 2006).

In the present study, we applied this approach to PTSD by examining different text types and subgroups of forum users. We compared language use in forum users with PTSD and people that went through trauma, but didn't develop PTSD, and general population data. The different subgroups of people with PTSD consisted of military veterans, police officers, firefighters, and civilians. We also accounted for the context of trauma (profession related trauma or personal life related trauma) and timespan (childhood trauma, adult life trauma, trauma within 12 months, and trauma years ago). As a control, we used groups comprising individuals without trauma or those who suffered trauma but developed no PTSD. We used linguistic markers of depression in language established originally by Pennebaker (Tausczik and Pennebaker, 2010) to examine correlations of PTSD with depression. Our results show a number of salient word use differences between subsets of PTSD sufferers, grouped by such factors as occupational category, nature of trauma and time elapsed since trauma. We also found that all PTSD groups had significant words usage differences relative to controls. 


\section{Methods}

\section{Data collection and categorization}

In this study we differentiate which forum users have PTSD from those that don't. Data for this study are composed of text samples from public forums collected and screened according to previously described procedures (Coppersmith et al., 2014a). Forums users often discuss their health for various reasons, such as to seek support or advice. More specifically to mental health, users may choose an anonymous forum because of social stigma associated with mental illness. Many forum users describe their diagnosis in a large variety of mental health conditions (Coppersmith et al., 2015). In this study we focused on PTSD. A human editor assessed each description of diagnosis and removed quotes, or other disingenuous text sections. To ensure that each included forum user has a sufficient amount of data, we ensured that each user had at least 100 words, however over $80 \%$ had between 200 and 500 words.

Ideally age and gender should be controlled for when performing mental health research. Only very few studies have taken matched samples into consideration. For example, by examining a specific subgroup of population such as college students (Rude et al., 2004). In order to have age- and gender-matched groups we analyzed each text sample and it's language as described in previous published studies (Sap et al., 2014). To obtain our final data set, for each user in the PTSD groups, we sampled paired control users of the same estimated gender with the closest estimate age.

\section{Data}

Text samples for the PTSD group were collected from various internet forums dedicated to PTSD (Tbl.1). A large number of possibly suitable text samples were manually screened and either discarded or sorted into the groups of interest based on the content of the excerpt itself, forum thread context and other available anonymous information. Previous studies often compare data from people affected by PTSD vs healthy people/general population data. In our study we also used general population data as a control group. However, it is also important to compare PTSD data to text samples from traumatized individuals that didn't develop PTSD to control for trauma-related word patterns unrelated to PTSD. This also allows for the possible detection of resilience-related word patterns. Therefore, our first control group consists of data from firefighter forums, where users discuss work, daily life and extreme/traumatic situations, 
but, however, are not PTSD sufferers (Tbl.1). Our second control group consists of general population data (Tbl.1).

We also distinguished between narrative related to the trauma and daily life events narratives (Tbl.1). Forums have various discussion group categorizations. For "trauma narratives" we choose text that discussed the trauma event explicitly. We collected text samples for "daily life narratives" from journal entry discussion groups where forum users shared their daily lives with other users but made no explicit mention of trauma events (Tbl.1).

Table 1

\begin{tabular}{|l|l|}
\hline Subgroup & $\begin{array}{l}\text { Number of text } \\
\text { samples (excerpts) }\end{array}$ \\
\hline Individuals with PTSD sharing daily events & 21 \\
\hline $\begin{array}{l}\text { Individuals who suffered trauma within last 12 month and are at } \\
\text { risk for PTSD }\end{array}$ & 16 \\
\hline Individuals who suffered trauma years ago and developed PTSD & 21 \\
\hline Military veterans, police officers, firefighters with PTSD & 19 \\
\hline Firefighters without PTSD (Control 1) & 19 \\
\hline General population (Control 2) & 26 \\
\hline
\end{tabular}

Datasets used for study (total $n=122$ ).

\section{Analysis}

We used word usage frequency analysis conceptually similar to that in LIWC methodology and program (Pennebaker et al., 2003). LIWC was shown to be effective in detecting a number of psychologically salient language usage patterns. We developed a custom software program that extended LIWC approach by combining it with character language models (CLMs) for additional word matching features as well as additional and/or modified word categories tailored to PTSD. This provided a score even for very short texts (McNamee, 2004). Word matching included pattern matching whole words, roots, salient word parts, simple stemming, split verb/expression stemming and others. 
Based on literature (Pennebaker, 1993; 2001; Stirman and Pennebaker, 2001; Pennebaker and Stone, 2003; Kenardy et al., 2007; Tausczik and Pennebaker, 2010; Badger et al., 2011; Jaeger et al., 2014; Mott et al., 2015; Knutsen and Jensen, 2017; Westerman et al., 2017; RometschOgioun El Sount et al., 2018) and text screening, the following word categories were determined to be potentially salient for PTSD and/or depression were used in this study:

- Singular first-person pronouns (related to self only)

- Plural first-person pronouns (related to group including self)

- Words positively correlated with depression(Stirman and Pennebaker, 2001; Pennebaker et al., 2003; Rude et al., 2004; Tausczik and Pennebaker, 2010; Baddeley et al., 2011; Mowery et al., 2017)

- Negative emotions

- Mortality, death and dying

- Indicators of cognitive complexity

- Words indicating causative relationships

For each word category and population group, a standard set of usage frequency statistical data was calculated (using stats and stats-lite npm software modules), including mean, median, variance, standard deviation, and percentile distribution. Statistical significance of group differences was calculated using t-test, ANOVA, and post hoc Tukey test. For each user, we scored each text based on the character n-grams in the text with the CLMs for the condition. This method followed previous work on predicting mental health in social media (Coppersmith et al., 2014a).

This study is an analysis of existing, de-identified and publicly available data. No sensitive information was collected, and the study DATA is completely anonymous. As by regulation of $\S 46.104$, if the project does not include any interaction or intervention with human subjects or include any access to identifiable private information, then the project does not require IRB review and is exempt.

\section{Results}

The present study employed a computerized text analysis to examine language usage patterns in people affected by PTSD. For research related to PTSD it is important to compare PTSD data to text samples from general population, but more so with data from people that went through 
trauma, and didn't develop PTSD in order to possibly detect resilience related word patterns. Our controls consisted of trauma narratives collected from firefighters that went through trauma but didn't develop PTSD (Control 1, N=19), and daily life narratives written by general population (Control 2, N=26). We analyzed online posts from forums related and unrelated to PTSD. We observed differences in linguistic markers in posts of similar word count written by different groups of people affected by PTSD $(\mathrm{N}=77)$ and two control groups.

\section{Texts from people with PTSD whose trauma occurred years ago differ from those written by PTSD sufferers with recent trauma}

We distinguished between people whose trauma occurred recently (within 12 months) and those who experienced trauma years ago, including childhood trauma. In both PTSD groups singular first-person pronouns usage was higher than in the two control groups (Mdiff $=0.068$, Mdiff $=$ 0.087; Mdiff = 0.061, Mdiff =0.08, p < 0.001, Fig. 1a). First person plural pronouns were lower in frequency in both PTSD groups compared to control 1, although in the group where trauma occurred years ago the usage was the lowest (Mdiff $=0.014$, Mdiff $=0.019, \mathrm{p}<0.001$, Fig. 1b).

Compared to control 2, only text from PTSD years ago group showed a significant difference in plural first-person pronoun usage (Mdiff =0.006; $\mathrm{p}>0.05$; Mdiff $=0.01, \mathrm{p}<0.05$, Fig.1b). Compared to control 1, the usage of negative emotion words was only different in the PTSD group where trauma occurred years ago (Mdiff $=0.0097, \mathrm{p}<0.001$, Fig. 1c). However, compared to control 2, both PTSD groups showed a significantly higher usage of negative emotion words (Mdiff $=0.0096, \mathrm{p}<0.001 ;$ Mdiff $=0.0149, \mathrm{p}<0.001$, Fig.1c). The usage of cognitive words was higher in both PTSD groups compared to control 1, but not control 2 (Mdiff $=0.038$, Mdiff $=0.049, \mathrm{p}<0.001 ;$ Mdiff $=0.003$, Mdiff $=0.007, \mathrm{p}>0.05$, Fig. $1 \mathrm{~d})$. In contrast to the previously published data (Jaeger, Lindblom, Parker-Guilbert, \& Zoellner, 2014), we could not detect a significant difference in causation words $(\mathrm{p}>0.05$, Fig.1e). Death related word usage was higher in both PTSD groups compared to control 2 (Mdiff $=0.0019, \mathrm{p}<0.05 ;$ Mdiff $=0.0023, \mathrm{p}<$ 0.001, Fig.1f).

\section{Figure 1}



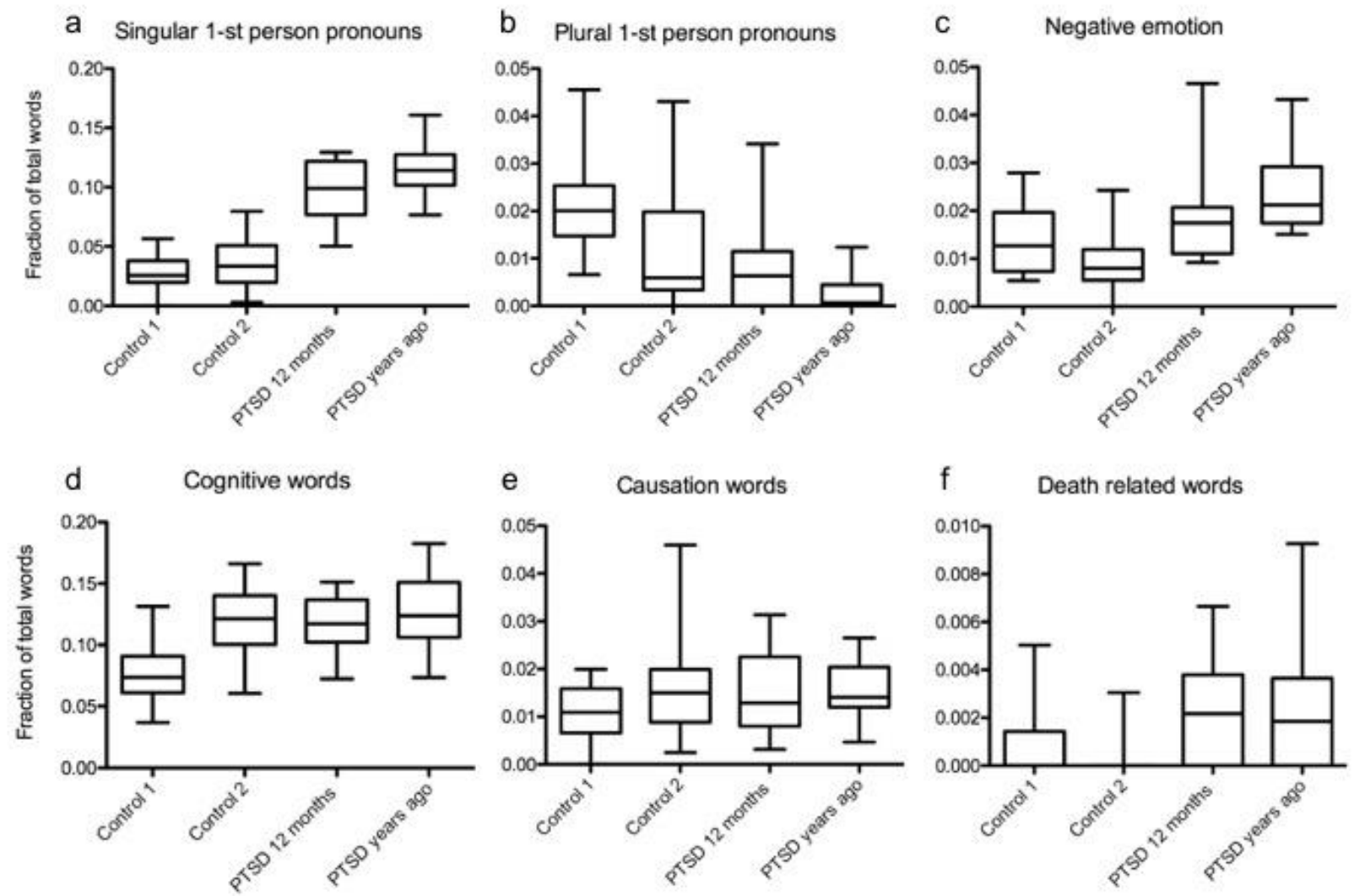

Fig. 1: Word usage patterns in texts related to trauma that occurred years ago and recent trauma. (a) Higher frequency of singular first-person pronouns in both PTSD groups compared to controls (Mdiff $=0.068$, Mdiff $=0.087$; Mdiff $=0.061$, Mdiff $=0.08, p<0.001$ ). (b) Lower frequency in plural first-person pronouns in both PTSD groups compared to control 1, the lowest one found in the group whose trauma occurred years ago (Mdiff $=0.014$, Mdiff $=0.019, \mathrm{p}<$ 0.001). Only PTSD group whose trauma occurred years ago showed a significant difference in plural first- person pronoun usage compared to control 2 (Mdiff $=0.006 ; p>0.05$; Mdiff $=0.01$, $\mathrm{p}<0.05$ ). (c) Usage of negative emotion words was only different from control 1 in the PTSD group where trauma occurred years ago (Mdiff $=0.0097, \mathrm{p}<0.001$ ). Compared to control 2, both PTSD groups showed a significantly higher usage of negative emotion words (Mdiff = $0.0096, \mathrm{p}<0.001$; Mdiff $=0.0149, \mathrm{p}<0.001$ ). (d) Cognitive words were more frequent in both PTSD groups compared to control 1, but not control 2 (Mdiff $=0.038$, Mdiff $=0.049, \mathrm{p}<0.001$; Mdiff $=0.003$, Mdiff $=0.007, \mathrm{p}>0.05$ ). (e) There was no significant difference in causation word usage between all 3 PTSD groups and/or controls ( $p>0.05$ ). (f) Death related word usage was higher in both PTSD groups compared to control 2 (Mdiff $=0.0019, \mathrm{p}<0.05$; Mdiff $=$ $0.0023, \mathrm{p}<0.001)$.

\section{Language usage differences detected comparing people with PTSD whose trauma occurred}

\section{in a work setting to PTSD sufferers with personal life related trauma}

We identified a set of variables in language usage in 2 different groups of PTSD, (1) people who went through trauma at work, including veterans, police officers, and firefighters (professional 
life), and (2) people that experienced trauma in their personal lives (civilians). The usage of singular first-person pronouns was higher in civilians, but also high in the professional group compared to both controls (Mdiff $=0.086$, Mdiff $=0.052, p<0.001$; Mdiff $=0.102$, Mdiff $=$ 0.0683, p < 0.001, Fig.2a). In both groups, plural first-person pronouns occurred significantly less frequently than in the control 1 . However, compared to control 2, only civilians affected by PTSD had a lower occurrence of plural first-person pronouns (Mdiff $=0.019, \operatorname{Mdiff}=0.015, \mathrm{p}<$ $0.001 ;$ Mdiff $=0.0103, \mathrm{p}<0.01$, Mdiff $=0.0068, \mathrm{p}>0.05$, Fig.2b). Negative emotion words were used more frequently in civilians compared to control 1 (Mdiff $=0.0097, \mathrm{p}<0.05$, Fig.2c). Compared to control 2, both PTSD groups had a higher frequency of negative emotion (Mdiff = 0.0149, $\mathrm{p}<0.001 ;$ Mdiff $=0.0086, \mathrm{p}<0.05$, Fig. 2c). Analysis of cognitive words showed a higher usage frequency in both PTSD groups than in control 1 (Mdiff $=0.049, \mathrm{p}<0.001$; Mdiff $=0.0503, \mathrm{p}<0.001$, Fig. $2 \mathrm{~d}$ ), but no difference compared to control 2 ( $\mathrm{p}>0.05$, Fig. $2 \mathrm{~d})$. There was no significant difference in causation word usage between the two PTSD groups and controls ( $p>0.05$, Fig. 2e). Death related word occurrence was higher in the professional life related trauma group than in both controls with the difference being greater when compared to control 2 (Mdiff = 0.0033, $p<0.05$; Mdiff $=0.004, p<0.001$, Fig. 2f).

\section{Figure 2}



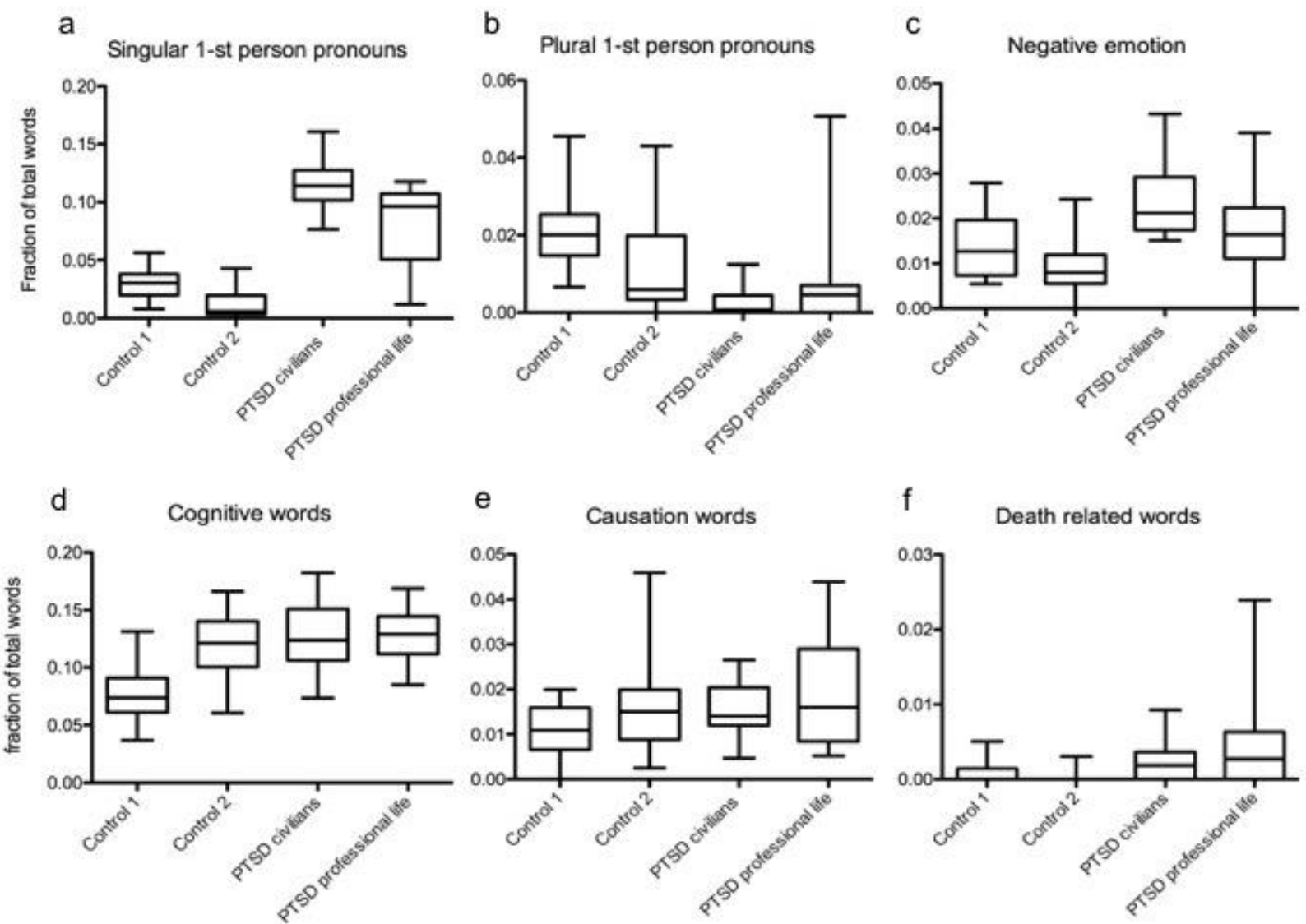

Fig. 2: Word patterns in texts from people PTSD sufferers whose trauma occurred in a work setting compared to personal life related trauma. (a) Higher frequency of singular firstperson pronouns in both PTSD groups compared to both controls, the highest found in civilians with PTSD $($ Mdiff $=0.086$, Mdiff $=0.052, \mathrm{p}<0.001 ;$ Mdiff $=0.102, \operatorname{Mdiff}=0.0683, \mathrm{p}<0.001$ ). (b) Lower frequency in plural first-person pronouns in both PTSD groups compared to control 1, but compared to control 2 only PTSD in civilians show a significant difference (Mdiff $=0.019$, Mdiff $=0.015, \mathrm{p}<0.001 ;$ Mdiff $=0.0103, \mathrm{p}<0.01$, Mdiff $=0.0068, \mathrm{p}>0.05$ ). (c) Both PTSD groups had a higher frequency of negative emotion words but only compared to control 2 (Mdiff $=0.0149, \mathrm{p}<0.001 ;$ Mdiff $=0.0086, \mathrm{p}<0.05$ ). Compared to control 1 only civilians showed a difference in negative emotion word usage (Mdiff $=0.0097, \mathrm{p}<0.05$ ). (d) Higher usage frequency of cognitive words in both PTSD groups compared to control 1 (Mdiff $=0.049, \mathrm{p}<$ 0.001 ; Mdiff $=0.0503, \mathrm{p}<0.001)$ but not to control $2(\mathrm{p}>0.05)$. (e) There was no significant difference in causation word usage between the two PTSD groups and/or controls ( $p>0.05)$. (f) Death related word occurrence was higher in the professional life related trauma group than in both controls, the difference being greater comparing to control 2 (Mdiff $=0.0033, p<0.05$; Mdiff $=0.004, \mathrm{p}<0.001)$.

Word patterns vary in different text types from people with PTSD: Comparing daily life narratives to trauma narratives 
We compared trauma narratives and daily life narratives from people with PTSD. In both PTSD related text types, singular first-person pronouns occurred more often than in controls. We found the highest frequency in the trauma narratives written by individuals whose trauma occurred years ago $($ Mdiff $=0.067$, Mdiff $=0.087$, Mdiff $=0.069, p<0.001 ;$ Mdiff $=0.0827$, Mdiff $=$ 0.102, Mdiff $=0.0844, \mathrm{p}<0.001$, Fig.3a). We detected a significantly lower usage of plural firstperson pronouns in daily narratives compared to recent trauma narratives (Mdiff $=0.0063, \mathrm{p}<$ 0.05, Fig.3b). Nevertheless, all 3 PTSD groups used fewer plural first-person pronouns than control 1, but compared to control 2 only the recent trauma group and daily narratives showed a significant difference $($ Mdiff $=0.019$, Mdiff $=0.014$, Mdiff $=0.02, p<0.001 ;$ Mdiff $=0.0069$, Mdiff $=0.0133, p<0.001$, Mdiff $=0.012, p>0.05$, Fig.3b). Negative emotion word usage was the highest in the daily narratives, but it was also higher than the controls in the narratives where trauma had occurred years ago. There was no significant difference in negative emotion word usage between recent trauma related texts and controls (Mdiff $=0.008, \mathrm{p}<0.05$; Mdiff $=0.0135$, $\mathrm{p}<0.05 ;$ Mdiff $=0.009, \mathrm{p}<0.05$, Mdiff $=0.015, \mathrm{p}>0.05$, Fig.3c). Cognitive word frequency was the highest in daily narratives of PTSD sufferers. In all three PTSD groups, it was higher than in control 1 but not significantly higher than in control 2 (Mdiff $=0.039$, Mdiff $=0.049$, Mdiff $=0.066, \mathrm{p}<0.001 ; \mathrm{p}>0.05$, Fig. 3d). There was no significant difference in causation word usage between PTSD groups and controls ( $\mathrm{p}>0.05$, Fig. 3e). Death related word usage was increased in both trauma text types compared to control 2 but not compared to control 1 (Mdiff = 0.0019 , Mdiff $=0.0023, p<0.05 ; p>0.05 ; p>0.05$, Fig. $3 f$ ).

\section{Figure 3}



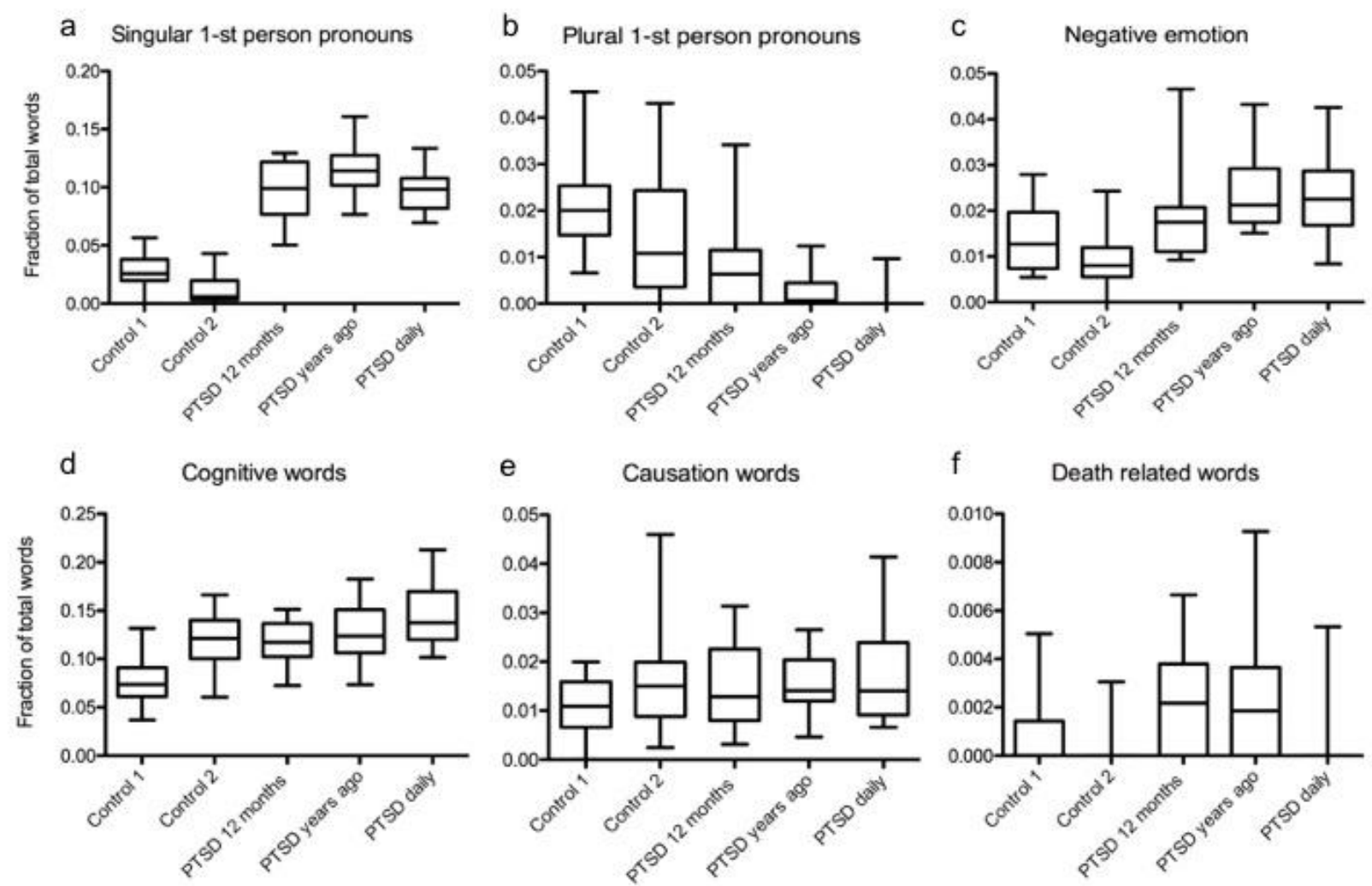

Fig. 3: Word usage patterns in daily narratives and trauma related narratives of PTDS sufferers. (a) Higher frequency of singular first-person pronouns in both PTSD related text types compared to controls, the highest one found in in the trauma narratives by individuals whose trauma occurred years ago $($ Mdiff $=0.067$, Mdiff $=0.087$, Mdiff $=0.069, \mathrm{p}<0.001$; Mdiff $=$ 0.0827 , Mdiff $=0.102$, Mdiff $=0.0844, p<0.001$ ). (b) Lower usage of plural first-person pronouns in daily narratives compared to recent trauma related text occurrence (Mdiff $=0.0063$, $\mathrm{p}<0.05$ ). However, all 3 PTSD groups used fewer plural first-person pronouns than control 1, but compared to control 2 only the recent trauma group and daily narratives showed a significant difference (Mdiff $=0.019$, Mdiff $=0.014$, Mdiff $=0.02, \mathrm{p}<0.001 ;$ Mdiff $=0.0069$, Mdiff $=$ $0.0133, \mathrm{p}<0.001$, Mdiff $=0.012, \mathrm{p}>0.05$ ). (c) Negative emotion word frequency was highest in daily narratives, but it was also high compared to controls in the reports of trauma that occurred years ago $($ Mdiff $=0.008, \mathrm{p}<0.05 ;$ Mdiff $=0.0135, \mathrm{p}<0.05$; Mdiff $=0.009, \mathrm{p}<0.05$, Mdiff $=$ $0.015, \mathrm{p}>0.05$ ). (d) Cognitive word frequency was the highest in daily narratives of PTSD sufferers. In all three PTSD groups, it was higher than in control 1, but not significantly higher than in control 2 (Mdiff $=0.039$, Mdiff $=0.049$, Mdiff $=0.066, p<0.001 ; p>0.05$ ). (e) There was no significant difference in causation word usage between PTSD groups and controls ( $p>$ 0.05). (f) Death related word type usage was increased in both trauma text types compared to only control 2 but not compared to control 1 , daily narratives didn't differ significantly compared to both controls $($ Mdiff $=0.0019, \operatorname{Mdiff}=0.0023, \mathrm{p}<0.05 ; \mathrm{p}>0.05 ; \mathrm{p}>0.05)$.

Language analysis suggests positive but variable correlation between depression and PTSD in different groups of affected people 
In this section of our study we used a recently developed procedure for analyzing depression through language usage, which is based on previous studies (Stirman \& Pennebaker, 2001).

We found the highest usage frequency of words positively correlated with depression in PTSD sufferers who experienced trauma years ago. However, the difference was only statistically significant compared to control 1, not to control 2 (Mdiff $=0.011, p<0.001 ; p>0.05$, Fig.4). The second highest usage frequency of depression-correlated words was found in the PTSD group with professional life related trauma (Mdiff $=0.007, \mathrm{p}<0.05$; Mdiff $=0.009, \mathrm{p}<0.05$, Fig.4). However, depression-correlated word usage was somewhat elevated in all PTSD groups we analyzed. Comparison of daily narratives from PTSD sufferers and daily narratives from the general population (control 2) points to an overall comorbidity of PTSD and depression as indicated by word usage (Mdiff $=0.0077, \mathrm{p}<0.01$, Fig.4).

\section{Figure 4}

Positive correlation to depression

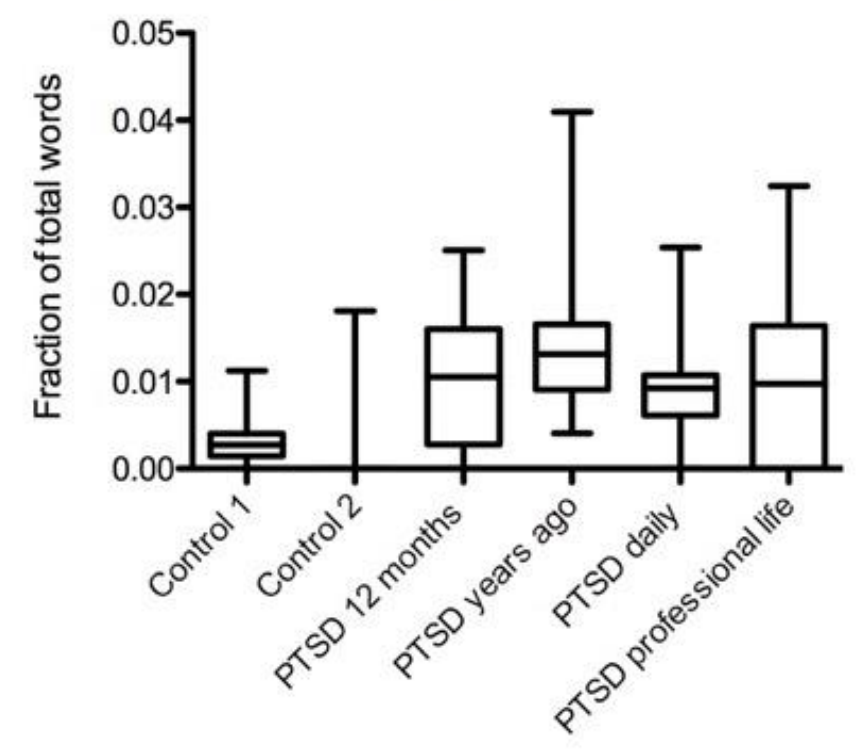

Fig. 4: Variable language-based positive correlation between depression and PTSD in different groups. The highest usage frequency of depression-correlated words was in PTSD sufferers who experienced trauma years ago, the difference being statistically significant compared to control 1, but not control 2 (Mdiff $=0.011$, Mdiff $=0.007, p<0.001, p>0.05$ ). The second highest usage frequency of depression-correlated words was in the PTSD group with professional life related trauma (Mdiff $=0.007$, Mdiff $=0.008, \mathrm{p}>0.05, \mathrm{p}<0.05$ ). Daily narratives of PTSD sufferers had more depression-correlated words than daily narratives of control 2, a general population sample (Mdiff $=0.0077, \mathrm{p}>0.05)$. 


\section{Discussion}

Social stigma along with self-presentation and self-assessment biases affect access to timely care either by preventing individuals with mental health conditions from seeking help or by reducing the accuracy of their evaluation by mental health professionals. To address this issue, researchers in mental health related fields started using implicit or indirect evaluation methods (Pressman and Cohen, 2007; Thoma et al., 2013). An important example of this, language analysis, may help to bypass self-presentation and self-assessment biases common in self reports (Mehl and Pennebaker, 2003; Pennebaker et al., 2003; Pennebaker, 2011). While a wide variety of semantic, syntactic and other language usage patterns may be associated with the state of a person's mental health, word usage frequencies appear to be the least affected by incidental variations. Some word categories appear to be especially significant for assessing an individual's mental state via language analysis. For instance, function words, such as pronouns, account for only about $1 \%$ of our total word usage, yet appear to be strongly linked to the underlying mental processes (Miller, 1995; Tausczik and Pennebaker, 2010).

Language usage, including word category frequencies, is affected by a variety of factors, including age (Pennebaker and Stone, 2003), gender (Mehl and Pennebaker, 2003), and personality (Hirsh et al., 2009). However, for certain word categories, the state of an individual's mental health may have the greatest impact on word usage frequency (Pennebaker, 1993).

Our data suggests that word usage in PTSD tends to differ from that of controls in a number of word categories, including first and third person pronouns, negative emotion words, death and dying related words, as well as words indicating cognitive complexity.

Furthermore, our data shows word usage differences between distinct groups of PTSD sufferers. We also found that word usage patterns are affected by the type of text. Overall, our results demonstrate the importance of taking into account additional factors, such as population subtypes and text types, when analyzing language usage as a reflection of mental states and their pathologies. Considering that lack of psychological support is a stronger predictor of PTSD that the severity or nature of the trauma (Perry et al., 1992), development of feasibly accurate and accessible analytical tools facilitating early diagnosis appears to have value for both prevention and effective treatment of PTSD.

We found the greatest differences in the usage of first person pronouns. All groups of PTSD sufferers, regardless of text type, had a markedly higher usage of singular first-person pronouns 
than the controls. This suggests an increased focus on oneself (Campbell and Pennebaker, 2003; Pennebaker et al., 2003), possibly at the expense of the focus on and collaboration with others. In fact, we found markedly lower usage of the plural first-person pronouns in text types of daily narratives and in civilians with PTSD whose trauma occurred years ago. On the other hand, individuals with recent trauma had an only mild-to-moderate decrease in plural first-person pronoun usage, which may reflect the dynamics of early stage PTSD development. Low occurrence of plural first-person pronouns in PTSD texts, particularly in long-standing PTSD, may also be an indicator of comorbidity with depression and a predictor of suicidal tendencies, which is consistent with the social integration model of suicide (Stirman and Pennebaker, 2001). The observed pattern of markedly higher singular first-person pronoun usage, especially when combined with lower frequency of the plural ones, may be salient not only for detecting existing PTSD but also, and perhaps more importantly, for assessing the risk of developing PTSD in individuals with recent trauma, i.e. during the time window when intervention and treatment tend to be the most effective. Studies show that the type of trauma is not, by itself, a reliable predictor of PTSD, whereas certain characteristic signs and symptoms, such as persistent avoidance, are (Dancu et al., 1996; Panasetis and Bryant, 2003; Briere et al., 2005). In that context, we should note the earlier findings that higher usage of singular first-person pronouns is associated with greater culpability and shame, which are predictive of developing PTSD during the first year post-trauma (Kubany et al., 2003; Negrao et al., 2005). Also, high singular first-person pronoun use in PTSD groups may be associated with trauma-related persistent dissociation, which is a strong predictor of PTSD (Briere et al., 2005). Overall, the observed pronoun usage patterns appear to have value for detecting both PTSD risk and onset.

We also found a moderately higher use of negative emotion words in all PTSD groups relative to controls. This word category is known to strongly correlate with the symptoms of depression. Notably, among PTSD groups, negative emotion words were higher in the groups with longstanding PTSD and lower (but still elevated relative to control) in the recent trauma group. We also analyzed the usage of depression-correlated words, a composite category comprising a representative sample of words commonly correlating with depression. We found that the usage of depression-correlated words was increased in all PTSD groups, but more dramatically in the group with long-standing PTSD. Our findings are consistent with prior research indicating that PTSD comorbidity with depression is common (ca. 50\%) and tends to occur in people with more 
severe and persistent forms of PTSD (Flory and Yehuda, 2015). This hypothesis appears to be further supported by our data showing higher death related word usage in the PTSD groups with either a very recent trauma or a trauma that occurred years ago. However, while the usage frequencies of death related words in these groups are elevated to similar levels, the reasons may be different. In the recent trauma group, the increase in death related word usage may reflect an acute reaction and processing of the recent trauma, especially if such trauma involved witnessing fatalities. In the group who experienced trauma far in the past, it may reflect a higher prevalence of severe, chronic depression that is linked to a higher risk of suicide (Stirman and Pennebaker, 2001). Proper interpretation of such group-related distinctions may improve the accuracy of prediagnostic evaluation and assist in guiding therapeutic intervention. Furthermore, it would be critical to recognize the subgroup of PTSD sufferers showing comorbidity with depression, because they generally have a higher level of neurocognitive distress (Blanchard et al., 1998; Nijdam et al., 2013; Flory and Yehuda, 2015) and are at a greater risk of suicide than people with PTSD alone (Campbell et al., 2007; Ramsawh et al., 2014). It would be also crucial to determine if current treatment options are effective in people showing comorbidity of PTSD with depression.

In our cognitive mechanism word analysis, we distinguished between causation and cognitive words. There were no significant differences in causation word frequencies between PTSD groups and controls. Cognitive word usage was increased in all PTSD groups relative to control 1 but was similar to control 2. This difference may be the most meaningful for the professional life related PTSD group for which control 1 (firefighters without PTSD) is a better match. Cognitive word usage was somewhat higher in the daily narratives of PTSD sufferers than in trauma narratives which is in line with previous research (Rubin, 2011).

It is essential for both research and clinical practice to be able to accurately assess psychological well-being in order to identify individuals who are at risk or have developed PTSD after trauma. Within that population, it is also critically important to detect cases at higher risk of suicide in view of a known relationship between traumatic experiences and suicidal behaviors (Knox, 2008).

Explicit screening methods are often deficient due to biases in self-assessment, self-presentation and self-reporting as well as avoidance due to lack of anonymity. Implicit screening methods, such as language analysis, have the capability to increase accuracy and reliability used either 
alone or in conjunction with explicit screening. Furthermore, they can provide anonymous selfpre-diagnosis without the risk of social stigma, thus reducing the obstacles to seeking immediate care. Facilitating prompt care could decrease PTSD rates or severity in people who have suffered a recent trauma. Further studies should help improve the accuracy and practical utility of language usage analysis for PTSD and other conditions. Such studies may involve further examining a variety of salient or confounding factors, such as different patient subgroups, situational context, co-morbid conditions and others. Analyzing language use patterns other than word frequencies, such as syntactic and semantic structures, may yield additional insights. Overall, as a supplementary tool, language analysis has an advantage of being cost and time efficient and also provides a convenient option for anonymous self-pre-diagnosis and screening.

\section{Author contributions statement}

Catarina Cunha and German Todorov conceived of the presented idea. German Todorov developed the theory and performed the computations. Catarina Cunha and Christopher Cain verified the analytical methods. Karthikeyan Mayilvahanan assisted German Todorov. All authors discussed the results and contributed to the final manuscript.

\section{Competing interests}

All author declare that they have no significant competing financial, professional, or personal interests that might have influenced the performance or presentation of the work described in this manuscript.

\section{Data availability statement}

The data that support the findings of this study are available from the corresponding author (G.T.), upon reasonable request.

\section{Acknowledgements}

We would like to thank Emma McBrian and David Ashurov for their help in categorizing the data. 
Acosta, J., Ramchand, R., Jaycox, L.H., Becker, A., and Eberhart, N.K. (2013). Interventions to Prevent Suicide: A Literature Review to Guide Evaluation of California's Mental Health Prevention and Early Intervention Initiative. Rand Health Q 2(4), 2.

Ahmedani, B.K. (2011). Mental Health Stigma: Society, Individuals, and the Profession. $J$ Soc Work Values Ethics 8(2), 41-416.

Alvarez-Conrad, J., Zoellner, L.A., and Foa, E.B. (2001). Linguistic predictors of trauma pathology and physical health. Applied Cognitive Psychology 15(7), S159-S170. doi: 10.1002/acp.839.

Baddeley, J.L., Daniel, G.R., and Pennebaker, J.W. (2011). How Henry Hellyer's use of language foretold his suicide. Crisis 32(5), 288-292. doi: 10.1027/0227-5910/a000092.

Badger, K., Royse, D., and Moore, K. (2011). What's in a story? A text analysis of burn survivors' web-posted narratives. Soc Work Health Care 50(8), 577-594. doi: 10.1080/00981389.2011.592114.

Baker, L., Wagner, T.H., Singer, S., and Bundorf, M.K. (2003). Use of the Internet and e-mail for health care information: results from a national survey. JAMA 289(18), 2400-2406. doi: 10.1001/jama.289.18.2400.

Berger, M., Wagner, T.H., and Baker, L.C. (2005). Internet use and stigmatized illness. Soc Sci Med 61(8), 1821-1827. doi: 10.1016/j.socscimed.2005.03.025.

Blanchard, E.B., Buckley, T.C., Hickling, E.J., and Taylor, A.E. (1998). Posttraumatic stress disorder and comorbid major depression: is the correlation an illusion? J Anxiety Disord 12(1), 21-37.

Boals, A., and Klein, K. (2005). Word Use in Emotional Narratives about Failed Romantic Relationships and Subsequent Mental Health. Journal of Language and Social Psychology 24(3), 252-268. doi: 10.1177/0261927x05278386.

Bonfils, K.A., Lysaker, P.H., Yanos, P.T., Siegel, A., Leonhardt, B.L., James, A.V., et al. (2018). Self-stigma in PTSD: Prevalence and correlates. Psychiatry Res 265, 7-12. doi: 10.1016/j.psychres.2018.04.004.

Booker, J.A., Graci, M.E., Hudak, L.A., Jovanovic, T., Rothbaum, B.O., Ressler, K.J., et al. (2018). Narratives in the Immediate Aftermath of Traumatic Injury: Markers of Ongoing Depressive and Posttraumatic Stress Disorder Symptoms. J Trauma Stress 31(2), 273 285. doi: 10.1002/jts.22271.

Brahm, P., Cortazar, A., Fillol, M.P., Mingo, M.V., Vielma, C., and Aranguiz, M.C. (2016). Maternal sensitivity and mental health: does an early childhood intervention programme have an impact? Fam Pract 33(3), 226-232. doi: 10.1093/fampra/cmv071.

Briere, J., Scott, C., and Weathers, F. (2005). Peritraumatic and persistent dissociation in the presumed etiology of PTSD. Am J Psychiatry 162(12), 2295-2301. doi: 10.1176/appi.ajp.162.12.2295.

Bucci, W., and Freedman, N. (1981). The language of depression. Bulletin of the Menninger Clinic 45(4), 334-358.

Burnam, M.A., Berry, S.H., Cerully, J.L., and Eberhart, N.K. (2014). Evaluation of the California Mental Health Services Authority's Prevention and Early Intervention Initiatives: Executive Summary and Commentary. Rand Health Q 4(1), 7.

Campbell, D.G., Felker, B.L., Liu, C.F., Yano, E.M., Kirchner, J.E., Chan, D., et al. (2007). Prevalence of depression-PTSD comorbidity: implications for clinical practice guidelines and primary care-based interventions. J Gen Intern Med 22(6), 711-718. doi: 10.1007/s11606-006-0101-4. 
Campbell, R.S., and Pennebaker, J.W. (2003). The secret life of pronouns: flexibility in writing style and physical health. Psychol Sci 14(1), 60-65. doi: 10.1111/1467-9280.01419.

Coppersmith, G., Dredze, M., and Harman, C. (2014a). Quantifying Mental Health Signals in Twitter. Conference Proceedings.

Coppersmith, G., Dredze, M., Harman, C., and Hollingshead, K. (2015). From ADHD to SAD: Analyzing the Language of Mental Health on Twitter through Self-Reported Diagnoses. Conference Proceedings.

Coppersmith, G., Harman, C., and Dredze, M. (2014b). Measuring Post Traumatic Stress Disorder in Twitter.

Dancu, C.V., Riggs, D.S., Hearst-Ikeda, D., Shoyer, B.G., and Foa, E.B. (1996). Dissociative experiences and posttraumatic stress disorder among female victims of criminal assault and rape. J Trauma Stress 9(2), 253-267.

Fisher, G.A., and Chon, K.K. (1989). Durkheim and the Social Construction of Emotions. Social Psychology Quarterly 52(1), 1-9. doi: 10.2307/2786899.

Flory, J.D., and Yehuda, R. (2015). Comorbidity between post-traumatic stress disorder and major depressive disorder: alternative explanations and treatment considerations. Dialogues Clin Neurosci 17(2), 141-150.

Griffiths, K.M., Calear, A.L., and Banfield, M. (2009). Systematic review on Internet Support Groups (ISGs) and depression (1): Do ISGs reduce depressive symptoms? J Med Internet Res 11(3), e40. doi: 10.2196/jmir.1270.

Hirsh, J.B., Deyoung, C.G., and Peterson, J.B. (2009). Metatraits of the Big Five differentially predict engagement and restraint of behavior. J Pers 77(4), 1085-1102. doi: 10.1111/j.1467-6494.2009.00575.x.

Horn, S.R., and Feder, A. (2018). Understanding Resilience and Preventing and Treating PTSD. Harv Rev Psychiatry 26(3), 158-174. doi: 10.1097/HRP.0000000000000194.

Houston, T.K., Cooper, L.A., and Ford, D.E. (2002). Internet support groups for depression: a 1year prospective cohort study. Am J Psychiatry 159(12), 2062-2068. doi: 10.1176/appi.ajp.159.12.2062.

Jaeger, J., Lindblom, K.M., Parker-Guilbert, K., and Zoellner, L.A. (2014). Trauma Narratives: It's What You Say, Not How You Say It. Psychol Trauma 6(5), 473-481. doi: 10.1037/a0035239.

Jones, N., Fear, N.T., Wessely, S., Thandi, G., and Greenberg, N. (2017). Forward psychiatry early intervention for mental health problems among UK armed forces in Afghanistan. Eur Psychiatry 39, 66-72. doi: 10.1016/j.eurpsy.2016.05.009.

Kaplow, J.B., Wardecker, B.M., Layne, C.M., Kross, E., Burnside, A., Edelstein, R.S., et al. (2018). Out of the Mouths of Babes: Links Between Linguistic Structure of Loss Narratives and Psychosocial Functioning in Parentally Bereaved Children. J Trauma Stress 31(3), 342-351. doi: 10.1002/jts.22293.

Kenardy, J., Smith, A., Spence, S.H., Lilley, P.R., Newcombe, P., Dob, R., et al. (2007). Dissociation in children's trauma narratives: an exploratory investigation. J Anxiety Disord 21(3), 456-466. doi: 10.1016/j.janxdis.2006.05.007.

Kessler, R.C., Berglund, P.A., Bruce, M.L., Koch, J.R., Laska, E.M., Leaf, P.J., et al. (2001). The prevalence and correlates of untreated serious mental illness. Health Serv Res $36(6 \mathrm{Pt}$ 1), 987-1007.

Knox, K.L. (2008). Epidemiology of the relationship between traumatic experience and suicidal behaviors. PTSD Research Quarterly 19(4)(Fall 2008). 
Knutsen, M., and Jensen, T.K. (2017). Changes in the trauma narratives of youth receiving trauma-focused cognitive behavioral therapy in relation to posttraumatic stress symptoms. Psychother Res, 1-13. doi: 10.1080/10503307.2017.1303208.

Kross, E., and Ayduk, O. (2008). Facilitating Adaptive Emotional Analysis: Distinguishing Distanced-Analysis of Depressive Experiences From Immersed-Analysis and Distraction. Personality and Social Psychology Bulletin 34(7), 924-938. doi: $10.1177 / 0146167208315938$.

Kubany, E.S., Hill, E.E., and Owens, J.A. (2003). Cognitive trauma therapy for battered women with PTSD: preliminary findings. J Trauma Stress 16(1), 81-91. doi: 10.1023/A:1022019629803.

Lampe, K., Doupi, P., and van den Hoven, M.J. (2003). Internet health resources: from quality to trust. Methods Inf Med 42(2), 134-142.

Lieberman, M.A., and Goldstein, B.A. (2006). Not all negative emotions are equal: the role of emotional expression in online support groups for women with breast cancer. Psychooncology 15(2), 160-168. doi: 10.1002/pon.932.

Link, B., Castille, D.M., and Stuber, J. (2008). Stigma and coercion in the context of outpatient treatment for people with mental illnesses. Soc Sci Med 67(3), 409-419. doi: 10.1016/j.socscimed.2008.03.015.

Lyons, E.J., Mehl, M.R., and Pennebaker, J.W. (2006). Pro-anorexics and recovering anorexics differ in their linguistic Internet self-presentation. J Psychosom Res 60(3), 253-256. doi: 10.1016/j.jpsychores.2005.07.017.

McNamee, P.M. (2004). Character N-Gram Tokenization for European Language Text Retrieval. J. Information Retrieval 7: 73.

Mehl, M.R., and Pennebaker, J.W. (2003). The sounds of social life: a psychometric analysis of students' daily social environments and natural conversations. J Pers Soc Psychol 84(4), 857-870.

Miller, G.A. (1995). Wordnet - a Lexical Database for English. Communications of the Acm 38(11), 39-41. doi: Doi 10.1145/219717.219748.

Mott, J.M., Galovski, T.E., Walsh, R.M., and Elwood, L.S. (2015). Change in Trauma Narratives and Perceived Recall Ability over a Course of Cognitive Processing Therapy for PTSD. Traumatology (Tallahass Fla) 21(1), 47-54. doi: 10.1037/trm0000012.

Mowery, D., Smith, H., Cheney, T., Stoddard, G., Coppersmith, G., Bryan, C., et al. (2017). Understanding Depressive Symptoms and Psychosocial Stressors on Twitter: A CorpusBased Study. J Med Internet Res 19(2), e48. doi: 10.2196/jmir.6895.

Negrao, C., 2nd, Bonanno, G.A., Noll, J.G., Putnam, F.W., and Trickett, P.K. (2005). Shame, humiliation, and childhood sexual abuse: distinct contributions and emotional coherence. Child Maltreat 10(4), 350-363. doi: 10.1177/1077559505279366.

Nijdam, M.J., Gersons, B.P., and Olff, M. (2013). The role of major depression in neurocognitive functioning in patients with posttraumatic stress disorder. Eur $J$ Psychotraumatol 4. doi: 10.3402/ejpt.v4i0.19979.

Norquist, G., and Hyman, S.E. (1999). Advances in understanding and treating mental illness: implications for policy. Health Aff (Millwood) 18(5), 32-47.

Olfson, M., Kessler, R.C., Berglund, P.A., and Lin, E. (1998). Psychiatric disorder onset and first treatment contact in the United States and Ontario. Am J Psychiatry 155(10), 1415-1422. doi: 10.1176/ajp.155.10.1415. 
Panasetis, P., and Bryant, R.A. (2003). Peritraumatic versus persistent dissociation in acute stress disorder. J Trauma Stress 16(6), 563-566. doi: 10.1023/B:JOTS.0000004079.74606.ba.

Pennebaker, J.W. (1993). Putting Stress into Words - Health, Linguistic, and Therapeutic Implications. Behaviour Research and Therapy 31(6), 539-548. doi: Doi 10.1016/00057967(93)90105-4.

Pennebaker, J.W. (2001). Dealing with a traumatic experience immediately after it occurs. Adv Mind Body Med 17(3), 160-162. doi: 10.1054/ambm.2000.0307.

Pennebaker, J.W. (2011). Your use of pronouns reveals your personality. Harv Bus Rev 89(12), 32-33.

Pennebaker, J.W., Mehl, M.R., and Niederhoffer, K.G. (2003). Psychological aspects of natural language. use: our words, our selves. Annu Rev Psychol 54, 547-577. doi:

10.1146/annurev.psych.54.101601.145041.

Pennebaker, J.W., and Stone, L.D. (2003). Words of wisdom: language use over the life span. $J$ Pers Soc Psychol 85(2), 291-301.

Perlick, D.A., Rosenheck, R.A., Clarkin, J.F., Sirey, J.A., Salahi, J., Struening, E.L., et al. (2001). Stigma as a barrier to recovery: Adverse effects of perceived stigma on social adaptation of persons diagnosed with bipolar affective disorder. Psychiatr Serv 52(12), 1627-1632. doi: 10.1176/appi.ps.52.12.1627.

Perry, S., Difede, J., Musngi, G., Frances, A.J., and Jacobsberg, L. (1992). Predictors of posttraumatic stress disorder after burn injury. Am J Psychiatry 149(7), 931-935. doi: 10.1176/ajp.149.7.931.

Pressman, S.D., and Cohen, S. (2007). Use of social words in autobiographies and longevity. Psychosom Med 69(3), 262-269. doi: 10.1097/PSY.0b013e31803cb919.

Pyszczynski, T., and Greenberg, J. (1987). Self-regulatory perseveration and the depressive selffocusing style: a self-awareness theory of reactive depression. Psychol Bull 102(1), 122138.

Ramsawh, H.J., Fullerton, C.S., Mash, H.B., Ng, T.H., Kessler, R.C., Stein, M.B., et al. (2014). Risk for suicidal behaviors associated with PTSD, depression, and their comorbidity in the U.S. Army. J Affect Disord 161, 116-122. doi: 10.1016/j.jad.2014.03.016.

Regier, D.A., Narrow, W.E., Rae, D.S., Manderscheid, R.W., Locke, B.Z., and Goodwin, F.K. (1993). The de facto US mental and addictive disorders service system. Epidemiologic catchment area prospective 1-year prevalence rates of disorders and services. Arch Gen Psychiatry 50(2), 85-94.

Rometsch-Ogioun El Sount, C., Windthorst, P., Denkinger, J., Ziser, K., Nikendei, C., Kindermann, D., et al. (2018). Chronic pain in refugees with posttraumatic stress disorder (PTSD): A systematic review on patients' characteristics and specific interventions. $J$ Psychosom Res. doi: 10.1016/j.jpsychores.2018.07.014.

Rubin, D.C. (2011). The coherence of memories for trauma: evidence from posttraumatic stress disorder. Conscious Cogn 20(3), 857-865. doi: 10.1016/j.concog.2010.03.018.

Rude, S., Gortner, E.-M., and Pennebaker, J. (2004). Language use of depressed and depressionvulnerable college students. Cognition and Emotion 18(8), 1121-1133. doi: 10.1080/02699930441000030.

Sap, M., Park, G.J., Eichstaedt, J.C., Kern, M.L., Stillwell, D., Kosinski, M., et al. (2014). Developing Age and Gender Predictive Lexica over Social Media. Conference Proceedings. 
Simmons, R.A., Gordon, P.C., and Chambless, D.L. (2005). Pronouns in Marital Interaction: What Do "You" and "I" Say About Marital Health? Psychological Science 16(12), 932936. doi: 10.1111/j.1467-9280.2005.01639.x.

Stirman, S.W., and Pennebaker, J.W. (2001). Word use in the poetry of suicidal and nonsuicidal poets. Psychosom Med 63(4), 517-522.

Tackman, A.M., Sbarra, D.A., Carey, A.L., Donnellan, M.B., Horn, A.B., Holtzman, N.S., et al. (2018). Depression, negative emotionality, and self-referential language: A multi-lab, multi-measure, and multi-language-task research synthesis. J Pers Soc Psychol. doi: $10.1037 / \mathrm{pspp} 0000187$.

Tausczik, Y.R., and Pennebaker, J.W. (2010). The Psychological Meaning of Words: LIWC and Computerized Text Analysis Methods. Journal of Language and Social Psychology 29(1), 24-54. doi: 10.1177/0261927x09351676.

Thoma, M.V., La Marca, R., Bronnimann, R., Finkel, L., Ehlert, U., and Nater, U.M. (2013). The effect of music on the human stress response. PLoS One 8(8), e70156. doi: 10.1371/journal.pone.0070156.

Weintraub, M., Taves, D.R., Hasday, J.D., Mushlin, A.I., and Lockwood, D.H. (1981). Determinants of response to anorexiants. Clinical Pharmacology \& Therapeutics 30(4), 528-533. doi: doi:10.1038/clpt.1981.198.

Westerman, N.K., Cobham, V.E., and McDermott, B. (2017). Trauma-Focused Cognitive Behavior Therapy: Narratives of Children and Adolescents. Qual Health Res 27(2), 226235. doi: 10.1177/1049732315627795. 